\title{
Personality Inventory for Children: A measure of biopsychosocial status in children and adolescents
}

\author{
Jo Magne Ingul ${ }^{\star, * *}$ \\ Hans M. Nordahl $I^{\star \star, \star \star *}$ \\ Jan Egil Wold* \\ Craig A. Olsson ${ }^{\star \star \star *}$ \\ * Department of Child and Adolescent \\ Psychiatry, Levanger Hospital, \\ Nord-Trøndelag Health Trust \\ ** Department of Psychology, Norwegian \\ University of Science and Technology, \\ NTNU, Trondheim \\ *** Department of Psychiatry, Levanger \\ Hospital, Nord-Trøndelag Health Trust, \\ Levanger \\ **** School of Psychology, Deakin \\ University; Murdoch Children's Research \\ Institute, Royal Children's Hospital and \\ the University of Melbourne
}

AUSTRALIA

NORWAY

\begin{abstract}
Background and Objectives: The purpose of this study was to investigate the discriminant and convergent validity of the Personality Inventory for Children revised version (PIC-R) with respect to DSM-IV Oppositional Defiant Disorder (ODD; $\mathrm{n}=21$ ), Generalized Anxiety Disorder (GAD; $\mathrm{n}=22)$ and non-patient controls $(\mathrm{NC} ; \mathrm{n}=42)$.

Methods: The study was a cross-sectional comparative study conducted in Trondheim, Norway, with children between 9-13 years old, referred to an university outpatient clinic.

Results: One way ANOVA with bonferroni post hoc test showed significant differences between ODD and GAD groups on 8 PIC-R scales, between the GAD and NC groups on 9 PIC-R scales and between ODD and NC groups on all PIC-R scales excluding the intellectual screening scale. Multigroup discriminant analysis (MDA) was performed to discriminate between the groups on a data driven basis. It identified two discriminant functions as important in differentiating between the three groups. These functions were able to correctly identify $92.2 \%$ of the cases.
\end{abstract}


Conclusions:The results indicate that specific PIC-R profiles have high discriminant and convergent validity for distinguishing GAD, ODD and NC groups. The PIC-R seems to be a useful screening tool in paediatric and neuropsychological settings.

Received: 14 December 2012

Revised: 9 December 2013

Accepted: 18 December 2013

\section{Background}

In paediatric care, use of mental health screening is scarce ${ }^{1}$. This is in contrast to the fact that (1) somatic disease significantly increases the risk of psychiatric disorders in children and adolescents ${ }^{2}$, and (2) that only one third $(36.2 \%)$ of children and adolescents are identified and in treatment for their mental health problem ${ }^{3}$, thus tools that are useful to screen the most common internalizing and externalizing disorders are needed. Children who have been classified into traditional psychiatric categories often display great heterogeneity in their presentation, and comorbidity is very common ${ }^{4}$. Egger, Costello, Erkanli \& Angold ${ }^{5}$ found that somatic complaints are strongly associated with emotional problems in girls and disruptive behaviour problems in boys; they recommend screening of all children and adolescents with persistent complaints of headaches, stomach aches, or musculoskeletal pains for psychiatric disorders. This calls for a careful and thorough assessment of children in paediatric and primary care, including a psychiatric evaluation. The use of mental health screening tools could substantially increase the number of children and adolescents identified as having a possible mental health problem $^{1}$, and hence aid the clinician in planning and implementing individually tailored treatment. To do this, measures that provide information on the psychopathological and psychosocial characteristics of children and their families are needed.
This requires that the screening tools or rating scales give reliable and valid information about the constructs they are meant to measure. Given that validity and reliability is established rating scales provide important information, including identification of problems along different behavioural dimensions and comparisons across applications, time and people ${ }^{6}$. Since most children in paediatric settings have different symptoms and degrees of symptoms, scales measuring several psychosocial domains and levels of symptomatology are recommended ${ }^{7}$.

The Personality Inventory for Children revised version (PIC-R) is a recognised and psychometrically validated dimensional assessment of a broad range of child psychopathology ${ }^{8-10}$. The PIC-R provides a multidimensional measure of childhood cognitive ability, family relations, social skills and the child's behaviours and emotions ${ }^{9,10}$. The PIC$\mathrm{R}$ has been used in a variety of settings to identify subtypes of psychopathology in children referred for neuropsychological assessment ${ }^{8}$, develop psychosocial profiles of alcohol-exposed children ${ }^{11}$, and to distinguish between conduct problems and emotionally disturbed students $^{12,13}$. As this tool has proven useful in many settings, its diagnostic specificity might be of importance in non specialised psychiatric settings. To date, there have been no studies investigating the ability of the PIC-R to differentiate between internalising and externalising behaviours related to DSM-IV diagnosis; yet to do so would enhance our understanding of 
these commonly used and influential descriptors of child behaviour.

The purpose of this study was to examine the discriminant and convergent validity of the Personality Inventory for Children revised version (PIC-R) with respect to DSM-IV Oppositional Defiant Disorder (ODD; $\mathrm{n}=21$ ), Generalized Anxiety Disorder (GAD; $n=22)$ and non-patient controls (NC; $n=42)$. GAD and ODD was chosen as diagnoses in the patient groups as they are the most common internalizing and externalizing disorders ${ }^{14,15}$, in addition they can be seen as opposites with respect to symptoms and problems. It was therefore expected that the response profiles would differ according to the salient features of each diagnosis. A measure of biopsychosocial status should reliably and validly be able to differentiate between these categories.

We predicted that the GAD group would have high scores on the anxiety scale, with moderately elevated scores on somatic concern, withdrawal and depression compared to the ODD and NC groups. The GAD group should also have low scores on the hyperactivity, and delinquency scales compared to the ODD group. In contrast, we predicted that the ODD group would have high scores on delinquency, and hyperactivity scales and show moderately elevated scores on the family relations and social skills scales compared to the GAD and NC groups. High scores on the hyperactivity scale were expected since the overlap and developmental continuity between ADHD and ODD is well established ${ }^{16}$, in sharing problems such as fidgetiness, over activity, difficulty conforming to rules and standards, and keeping to routines. We expected both the GAD and the ODD groups to have high scores on the adjustment scale compared to the NC group. To determine whether the three groups can be differentiated on the basis of PIC-R factors, a discriminant analysis was performed.

\section{Method}

\section{Design \& procedure}

The study was approved by the Data Inspectorate, the committee for medical research ethics and conducted according to the ethical principles of APA (APA, 2002). The research design was a cross-sectional comparative study of three groups of children aged between 9-13 years. Data were collected using self-report measures and clinical interviews. Following referral from the Child and Family Guidance Clinic, each child and his/her parents were asked to attend an assessment of the child's behavior and to establish a diagnosis.

A multi-method and multi-informant strategy was used that included ratings and information from parents, children, teachers, and clinicians. Parents were also asked to complete a self-report battery while the child was assessed in a clinical interview. The research team interviewed teachers and conducted the screening of the non-patient controls at the various schools. Further details on assessment procedures are provided in two previously published papers ${ }^{17,18}$.

\section{Participants}

Seventy-five children recruited from five child and family guidance clinics were assessed at an outpatient clinic for the treatment of conduct or anxiety disorders. Inclusion criteria were developed to secure homogeneity of the diagnostic groups. First, each child had to be between 9 and 13 years of age and second, the ODD or GAD diagnosis had to be the child's primary problem of referral. Exclusion criteria were the presence of any other primary psychiatric disorder including reading and writing disabilities, mental or cognitive disabilities and ADHD. 
Of the 75 recruited children, 32 were excluded because they either did not meet the criteria for GAD or ODD diagnosis $(n=21)$ or their parents refused to participate $(n=11)$. The remaining forty-three were included in the study (GAD $n=21$, ODD $n=22)$. The nonpatient control group consisted of students from an age- and gender-matched classroom control with no symptoms of either behaviour disorders or anxiety problems. Of the 50 families asked to participate in the NC group, 42 gave their consent.
The total number of participants was 85 . Of these, $59 \%$ were boys and $41 \%$ were girls. The overall mean age was $10.2(\mathrm{SD}=1.3)$; for the GAD group the mean age was 9.9 years, for the ODD group 10.1 and for the non-patient controls 10.3. Most of the participants were Caucasian ( $85 \%$ ). $25 \%$ were in a low income condition, $34 \%$ were medium and $23 \%$ were high. There were no significant differences between the groups with regards to age, race, marital status or income. There was, however, a non-significant ten-

Table 1

Demographic factors for the children in the three groups

\begin{tabular}{|c|c|c|c|c|c|c|c|}
\hline \multirow[b]{2}{*}{ Characteristic } & \multicolumn{2}{|c|}{$\operatorname{ODD}(\mathrm{N}=21)$} & \multicolumn{2}{|c|}{ GAD $(N=22)$} & \multicolumn{2}{|c|}{$\mathrm{NC}(\mathrm{N}=42)$} & \multirow[b]{2}{*}{$\mathrm{p}$} \\
\hline & $\mathrm{N}$ & $\%$ & $\mathrm{~N}$ & $\%$ & $\mathrm{~N}$ & $\%$ & \\
\hline Gender & & & & & & & 0.01 \\
\hline Boy & 18 & 85.7 & 12 & 54.5 & 20 & 47.6 & \\
\hline Girl & 3 & 14.3 & 10 & 45.5 & 22 & 52.4 & \\
\hline Age & & & & & & & 0.79 \\
\hline 8-9 & 7 & 33.3 & 9 & 40.9 & 12 & 28.5 & \\
\hline $10-11$ & 11 & 52.3 & 10 & 45.4 & 22 & 52.1 & \\
\hline $12-13$ & 3 & 14.4 & 3 & 13.7 & 9 & 21.4 & \\
\hline Race & & & & & & & 0.22 \\
\hline Caucasian & 16 & 76.2 & 18 & 81.9 & 38 & 90.4 & \\
\hline African & 3 & 14.3 & 3 & 13.6 & 3 & 7.2 & \\
\hline Asian & 0 & 0.0 & 1 & 4.5 & 1 & 2.4 & \\
\hline Hispanic & 2 & 9.5 & 0 & 0.0 & 0 & 0.0 & \\
\hline \multicolumn{8}{|l|}{ Parents } \\
\hline Marital Status & & & & & & & 0.63 \\
\hline Married/ cohabitant & 12 & 57.2 & 15 & 68.2 & 29 & 69.0 & \\
\hline Single & 7 & 33.3 & 5 & 22.7 & 6 & 23.8 & \\
\hline No response & 2 & 9.5 & 2 & 9.1 & 3 & 7.2 & \\
\hline Family Income (SES) & & & & & & & 0.10 \\
\hline Low & 6 & 28.5 & 6 & 27.3 & 8 & 19.0 & \\
\hline Medicore & 5 & 23.8 & 6 & 27.3 & 12 & 28.6 & \\
\hline High & 7 & 33.3 & 8 & 36.3 & 14 & 33.3 & \\
\hline No response & 3 & 14.4 & 2 & 9.1 & 8 & 19.1 & \\
\hline
\end{tabular}

Note: Abbreviations: ODD= Oppositional Defiant Disorder; GAD = Generalised anxiety Disorder; NC $=$ nonpatient controls. 
dency in the ODD group $(\mathrm{p}<.10)$ to be in the low income condition. There were more boys in the ODD group (83\%) compared with the GAD group $(48 \%)\left(\mathrm{U}=147, \mathrm{~N}_{1}=21, \mathrm{~N}_{2}=\right.$ $22, \mathrm{p}=0.02$, two tailed). All participants answered the PIC-R, however 7 had to be discarded as invalid due to missing responses.

\section{Assessment}

The children and their parents, mostly mothers, were interviewed separately. The children were interviewed and assessed according to the DSM-IV criteria for axis I diagnosis ${ }^{15}$, using a semi-structured interview, where the DSM-IV criteria for childhood GAD and ODD were used. In addition, they were asked questions about difficulties related to friends, school, family disruption and general adjustment. Diagnostic assessments were conducted in three different settings. First, the child counsellor at the Child and Family Guidance Clinic screened and referred the children for treatment. Secondly, the principal investigator assessed the children according to DSM-IV disorders. Finally, to evaluate the reliability of ODD or GAD diagnoses, 15 GAD interviews and 15 ODD interviews were audio- or videotaped and independently rated by an external clinical psychologist. Kappa for the ODD and GAD diagnoses were 0.93 and 0.84 , respectively.

\section{Instruments}

The revised version of PIC-R ${ }^{9,10}$ was used. The inventory is completed by either the child's mother or father. The PIC-R contains 420 items answered in a true/false format. Interpretation and clinical use of the PIC-R are based on empirically replicated correlates between specific scale elevations and independent measures of the child status. Scores were obtained for 13 profile scales; 1 general screening scale -
Adjustment (ADJ); and 12 clinical scales Achievement (ACH), Intellectual screening (IS), Development (DVL), Somatic concern (SOM), Depression (D), Family relations (FAM), Delinquency (DLQ), Withdrawal (WDL), Anxiety (ANX), Psychosis (PSY), Hyperactivity (HPR), and Social skills (SSK).

The PIC-R has norms for both girls and boys between the ages of 3 to 5 and 6 to 16 , with the exception of the IS scale which is normed for ages 3-5, 6, 7, 8, 9 and 10-16. For all scales, raw scores are converted into $\mathrm{T}$ scores, where increases in $\mathrm{T}$ reflect greater levels of psychopathology. The PIC-R has good psychometric properties, with an internal consistency ranging from 0.57 to 0.86 and an average on the 13 clinical scales of $0.74^{9}$. Test-retest reliability coefficients for the clinical scales are 0.86 for a two-week interval and 0.71 for a two month interval, although crossinformant reliability is at the lower end of what is acceptable, according to the results of two clinical populations showing an average correspondence of 0.69 and $0.66^{9}$.

\section{Statistical analysis}

Chi square tests and Mann-Whitney tests were used to compare the groups on demographic variables. A one way ANOVA analysis with the Bonferroni post-hoc test was used to compare NC, GAD and ODD groups on PIC-R scales. Multigroup discriminant analysis (MDA) was then performed to test whether the groups could be separated on the basis of data driven factors. MDA combines independent variables that classify groups and is appropriate to use when assessing which factors are the best indicators of separation between groups. MDA is based on factor analytic methods that identify sets of variables that are powerful in discriminating between groups of subjects on a data-driven basis. The MDA is typically a one-way 
analysis and no problems are posed by unequal sample sizes in groups. The number of participants in the GAD and ODD clinical groups exceeded the number of predictor variables 2-fold, indicating sufficient sample size for MDA analysis ${ }^{20}$. Statistical analysis was performed using SPSS 18.

\section{Results}

Characteristics of the sample and demographic results are shown in Table 1. The PIC$\mathrm{R}$ profiles for each group are shown in figure 1 and table 2. The ODD group showed high scores on the adjustment, and delinquency

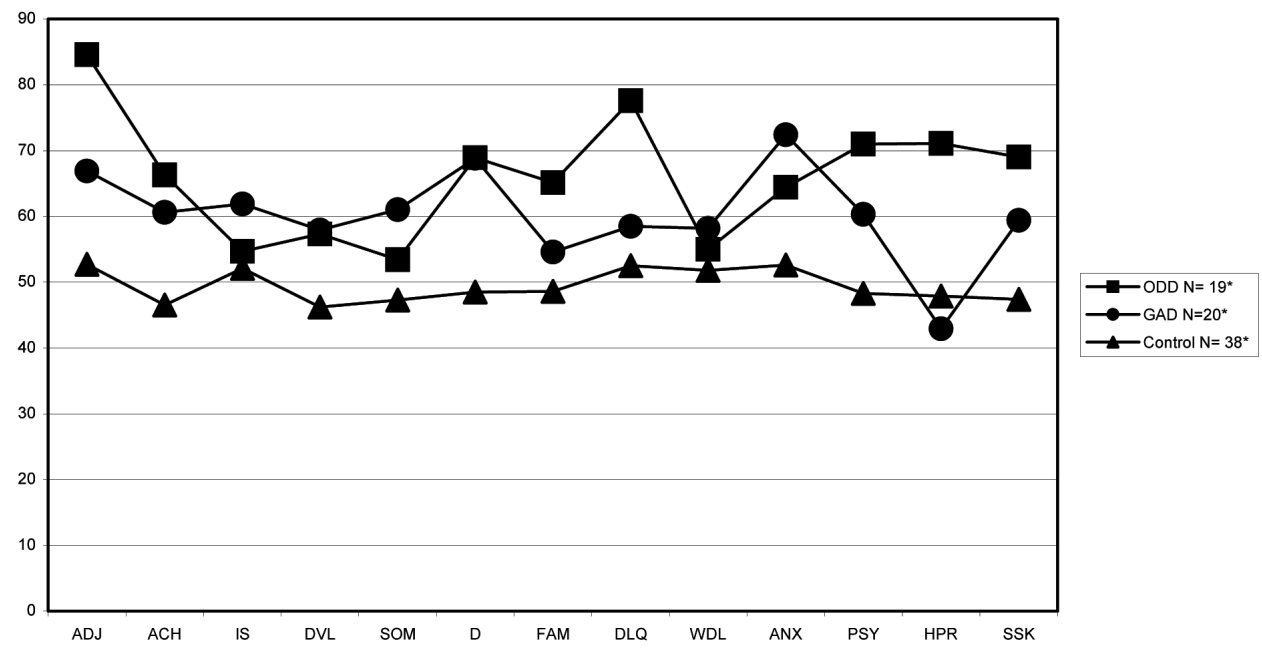

Figure 1. PIC-R profiles for the clinical scales for the ODD, GAD and NC groups.

Note: Abbreviations: $A D J=$ Adjustment, $A C H=$ Achievement, $I S=$ Intellectual screening, $D V L=$ Development, SOM = Somatic Concern, $D=$ Depression, FAM = Family Relations, $D L Q=$ Delinquency, $W D L=$ Withdrawal, ANX $=$ Anxiety, $P S Y=$ Psychosis, $H P R=$ Hyperactivity, $S S K=$ Social skills.

* Note: Due to missing responses on the PIC-R, 2 profiles in each clinical group and 4 in the Normal Control group were discarded as invalid.

scales, with moderately elevated scores on the family relations, hyperactivity, psychosis, achievement and depression scales. There were no low scores in the ODD profile. The GAD group scored high on anxiety and was moderately elevated on the adjustment, somatic concern and depression scales. The GAD group had low scores on the hyperactivity scale. For the NC group, all clinical scales were within the normal range.

ANOVA (with the Bonferroni post hoc test) indicated significant between group differences in PIC-R response profiles (see Table 2). There were significant differences between the GAD group and the ODD group on seven PIC-R scales. On all scales except for anxiety, ODD had a higher mean score, indicating greater problem interference. There were significant differences between the GAD group and the non-patient controls on eight clinical scales, and the adjustment scale all of which show greater problems for the GAD group. Between the ODD group and the non patient controls all clinical scales were significantly different except the intellectual screening scale, somatic concern scale and the withdrawal scale. All of these indicate greater problems for the ODD group. 
Table 2

PIC-R clinical scales

\begin{tabular}{|c|c|c|c|c|c|c|}
\hline & ODD $(\mathrm{N}=19)$ & $\mathrm{GAD}(\mathrm{N}=20)$ & Control $(\mathrm{N}=3$ & & & \\
\hline & $\mathrm{M}(\mathrm{SD})$ & $\mathrm{M}(\mathrm{SD})$ & $\mathrm{M}(\mathrm{SD})$ & $\mathrm{F}$ & $\mathrm{df}$ & $\mathrm{p}$ \\
\hline Adjustment & $84.6(12.4)$ & $66.9(9.6)$ & $52.7(5.6)$ & 84.69 & 2.74 & $0.001^{* 1.2 .3}$ \\
\hline Achievement & $66.3(11.6)$ & $60.6(8.8)$ & $46.5(7.3)$ & 36.69 & 2.74 & $0.001^{* 2.3}$ \\
\hline Intellectual screening & $54.7(18.6)$ & $61.9(20.9)$ & $52.1(10.4)$ & 2.49 & 2.74 & 0.090 \\
\hline Development & $57.3(12.3)$ & $57.9(10.9)$ & $46.2(7.8)$ & 12.81 & 2.74 & $0.001^{* 2.3}$ \\
\hline Somatic concern & $53.4(8.0)$ & $61.0(15.1)$ & $47.3(6.9)$ & 12.69 & 2.74 & $0.001^{* 3}$ \\
\hline Depression & $68.9(11.1)$ & $68.8(10.8)$ & $48.5(7.6)$ & 44.76 & 2.74 & $0.001^{* 2.3}$ \\
\hline Family relation & $65.1(12.3)$ & $54.6(9.6)$ & $48.6(7.2)$ & 19.92 & 2.74 & $0.001^{* 1.2}$ \\
\hline Delinquency & $77.6(11.0)$ & $58.5(12.0)$ & $52.5(5.9)$ & 48.12 & 2.74 & $0.001^{* 1.2}$ \\
\hline Withdrawal & $55.0(12.3)$ & $58.2(10.9)$ & $51.8(5.8)$ & 3.23 & 2.74 & $0.045^{* 3}$ \\
\hline Anxiety & $64.4(10.1)$ & $72.4(10.5)$ & $52.6(9.0)$ & 29.12. & 2.74 & $0.001^{* 1.2 .3}$ \\
\hline Psychosis & $71.0(16.2)$ & $60.3(15.5)$ & $48.3(7.7)$ & 21.86 & 2.74 & $0.001^{* 1.2 .3}$ \\
\hline Hyperactivity & $71.1(8.1)$ & $42.9(9.9)$ & $47.9(6.1)$ & 76.96 & 2.74 & $0.001^{* 1.2}$ \\
\hline Social Skills & $69.0(12.7)$ & $59.4(9.8)$ & $47.4(7.3)$ & 34.51 & 2.74 & $0.001^{* 1.2 .3}$ \\
\hline
\end{tabular}

Note: $* 1=$ significant between ODD and GAD group. $2=$ Significant between ODD- and NC. $3=$ Significant between GAD and NC.

\section{Multigroup discriminant analysis}

Results of the discriminant analysis are shown in table 3. The MDA was performed using the diagnostic groups to define membership and the PIC-R scales as discriminating variables.

Two discriminant functions were identified with a combined Wilks' lambda $=0.071, \chi^{2}$ $(26)=179,86, p<0.001$. After removal of the first function, there was still a significant association between predictors and groups (Wilks' lambda $=0.469 \chi^{2}(12)=51,5, \mathrm{p}<$ 0.001 ), indicating that both functions were informative in discriminating between the groups. Table 3 shows that adjustment; hyperactivity and delinquency had the highest loadings on function 1. This function contrasts socialised, healthy behaviours with im- pulsive, hyperactive, unsocialised behaviour. Anxiety, depression and somatic concern had the highest loadings on function 2 , which contrasts an introverted, emotional, unstable style with and an active, emotional stable style. These discriminant functions correctly classified $92.2 \%$ of the cases in the three groups. They correctly predicted $89.5 \%$ of the ODD group, whilst they placed $10.5 \%$ in the GAD group. For the GAD group they correctly predicted $85 \%$ of the cases, placing $15 \%$ in the $\mathrm{NC}$ group. For the NC group they predicted 97.4\%, correctly placing $2.6 \%$ in the GAD group. Group centroids were plotted as a discriminant perceptual map (see figure 2) to provide a visual representation of group differences with respect to the two discriminant functions. 
Table 3

Correlation between discriminant functions and PIC-R predictors for the GAD. ODD and NC groups

\begin{tabular}{lcc} 
& Discriminant & Function \\
\hline Predictor variable & 1 & 2 \\
\hline Adjustment & $0.622^{*}$ & 0.331 \\
Hyperactivity & $0.556^{*}$ & -0.554 \\
Delinquency & $0.482^{*}$ & 0.015 \\
Social skills & $0.383^{*}$ & 0.312 \\
Psychosis & $0.308^{*}$ & 0.230 \\
Family relations & $0.307^{*}$ & 0.103 \\
Anxiety & 0.175 & $0.737^{*}$ \\
Depression & 0.345 & $0.691^{*}$ \\
Somatic concern & 0.086 & $0.516^{*}$ \\
Achievement & 0.368 & $0.452^{*}$ \\
Development & 0.177 & $0.387^{*}$ \\
Withdrawal & 0.048 & $0.256^{*}$ \\
Intellectual screening & 0.016 & $0.241^{*}$ \\
\hline Canonical R & 0.921 & 0.729 \\
Eigenvalue & 5.603 & 1.133 \\
\hline
\end{tabular}

Note: * Largest absolute correlation between each variable and any discriminant function.

\section{Discussion}

The results of this study suggest that scales of the PIC-R are capable of differentiating salient behavioural features in carefully diagnosed samples of children having either a GAD or an ODD diagnosis. Between the two groups, PIC-R represented ODD children as being more delinquent, active and chaotic in their behaviour, showing poor social skills. They also had more family problems than the GAD children and the NC children. The only clinical scale on which the GAD group showed significantly greater problems than the ODD group was the anxiety scale. Compared to the NC group, the GAD children showed signifi- cantly greater problems on all the scales representing internalizing behaviours. Both diagnostic groups had elevations on the adjustment scale compared to the $\mathrm{NC}$ group.

Furthermore, results from the discriminant analysis showed that the groups can be differentiated successfully on the basis of a twofactor solution. The first factor contrasts socialised, healthy behaviour with hyperactive, unsocialised behaviour. The second factor contrasts an emotional, unstable, neurotic style with an active, emotional stable style. "Active" in this instance is seen as positively or socialised active rather than unsocialised active or hyperactive. The two discriminant functions were highly successful and sensitive in predicting group membership. Overall, the analysis predicted between $97.4 \%$ and $85 \%$ of the cases correctly depending on group membership.

For the ODD group, these findings are consistent with other research findings. Greene $e t$ $a l .{ }^{16}$ found that children with ODD have social and familial adjustment problems. They show poor social skills and have great difficulty interacting with other children compared to a normal control group and a psychiatric comparison group. Nordahl, Ingul, Nordvik \& Wells ${ }^{17}$ showed that ODD and GAD in children are differently related to their mothers' psychopathology and that ODD in children are related to mothers' expression of negative emotions and detached personality styles, indicating greater family conflict and hostility. On the PIC-R, the ODD children show a pattern of emotional instability, interaction problems and a chaotic, hyperactive norm-breaking behaviour compared to the NC group. Compared with the DSM IV criteria $^{19}$, the PIC-R profiles in this study seem to fit well. DSM IV Criteria A for ODD states that the child must show a pattern of negativistic, hostile and defiant behaviour, which fits well with elevated scores on the 


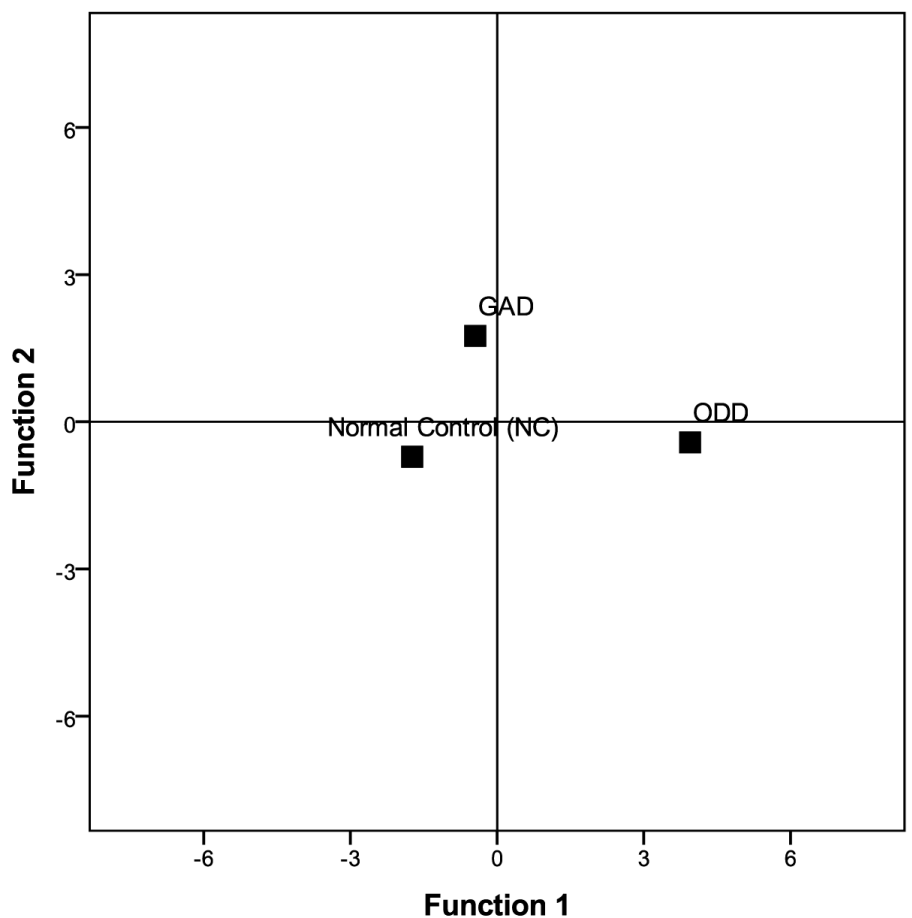

Figure 2. Plots of three group centroids on two discriminant functions derived from PIC-R predictors. Function 1 represents socialised vs. unsocialised behaviour.

Function 2 represents emotional unstable/ neurotic style vs. emotional stable and active style.

delinquency, psychosis, and hyperactivity scales. Elevated scores on the adjustment and social skills scales fit well with the impairment criteria (B) in DSM IV. Criteria C, which implies that the behaviours do not occur exclusively during the course of psychotic or mood disorder, is not possible to evaluate accurately using the PIC-R. However, the depression scale was elevated for the ODD group compared to the NC group, indicating symptoms of depression. Only $26.3 \%$ had scores higher than the actuarial guidelines, indicating that this is not a result of major depression.

The differences between the GAD group and the NC group also seem to be theoretically meaningful and consistent with other research findings ${ }^{21}$. The PIC-R profile of GAD children are, compared to normal controls, emotionally unstable, worried, somatically concerned, withdrawn, preoccupied with anxiety and show greater problems adjusting and handling their developmental age-appropriate tasks. Consistent with this, Warren and Sroufe $^{22}$ found that anxiety compromises development. Compared to DSM IV Criteria for GAD, the PIC-R profiles fit well. The elevated scores on anxiety, somatic concerns, depression and psychosis scales in the PIC$\mathrm{R}$ profiles fit well with the A-D criteria of DSM IV. Criteria E is the impairment criteria required for a GAD diagnosis; this fits well with the elevated scores on the adjustment scale of the PIC-R. The PIC-R does not take into account the length of time that both disorders must have been present ( 6 months) 
to set the diagnosis. All in all, it seems reasonable to say that the PIC-R shows good convergent and discriminant validity for the GAD and ODD diagnoses.

GAD and ODD can be seen as opposites with respect to symptoms and problems. The PIC-R factors in the discriminant analysis seem to pinpoint these differences. Although there were very few false positives in the analysis, the pattern of these is interesting and shows that the functions have limitations. They may point to a dimensional nature of psychopathology, or may indicate a continuum in the development of childhood psychopathol$o g y^{23}$. The findings on the discriminant analysis support the results of the ANOVA: the PIC-R is able to differentiate between the GAD and ODD children, and it gives salient information consistent with research findings and diagnostic requirements about the different diagnoses.

There are methodological limitations to this study. Our data were predominantly collected from the children and their mothers. A better representation of fathers as respondents could give a broader description of the children. For ODD and other externalising disorders, however, a significant overlap between information gathered from mothers and other reports has been shown ${ }^{24}$, while for GAD and other anxiety disorders only moderate agreement between parental reports of their children's anxiety has been shown ${ }^{25}$. The PIC-R has in itself some weaknesses worth considering. First of all it has a lot of items that has to be answered, and similar instruments as for instance the $\mathrm{CBCL}^{26}$ could be considered as a good alternative. However, Pearson and Lachar $^{27}$ have shown that the PIC-R has a greater ability to accurately identify children with adequate versus inadequate behaviour than the CBCL( $82-86 \%$ vs $73 \%$ ). Secondly, two of the subscales of the instruments (Intellectual screening and achie- vement) has internal consistency at the lower end of what is acceptable, indicating that these subscales has to be interpreted with caution. The present sample size is also relatively small. Although the statistical power is adequate to perform MDA analysis, it runs the risk of creating an overfitting effect, which could have implications for generalisation ${ }^{20}$.

\section{Conclusions}

Keeping these limitations in mind, it seems that the PIC-R has good discriminant and convergent validity for the various groups of ODD, GAD and NC. It captures the salient features of the disorders and could aid clinicians in setting accurate diagnoses. The MDA analysis gives further information on important aspects of the PIC-R profiles that should be carefully analysed when assessing children and adolescents. Therefore, still bearing the limitations in mind, it seems that the PIC$\mathrm{R}$ can be recommended as a useful, reliable and valid tool of psychopathology in paediatric and neuropsychological settings. PIC-R is tied to DSM categories in children, thereby potentially increasing the number of patients being identified with mental health problems alongside their paediatric problems if used in non specialised settings.

\section{Acknowledgement}

The authors would like to thank employees at the Child and adolescent psychiatry department at Levanger Hospital, and employees in primary health care in Nord-Trøndelag county for help in recruiting and assessing the children. The study was funded by the Liaison Committee between the Central Norway 
Regional Health Authority (RHA) and the Norwegian University of Science and Technology (NTNU) (2010/14279).

\section{Authors contributions}

Jo Magne Ingul performed statistical analysis and prepared the draft. Hans Nordahl designed the study and supervised the data collection. Jan Egil Wold and Craig A. Olsson helped to draft the manuscript. All authors read and approved the manuscript.

\section{References}

1. Brown JD, Wissow LS. Screening to identify mental health problems in pediatric primary care: Considerations for practice. Int J Psychiatry Med 2010; 40: 1-19.

2. Sandberg S, Stevenson J. Psychiatric aspects of somatic disease. In: Rutter M, Bishop DVM, Pine DS, Scott S, Stevenson J, Taylor E et al., eds. Rutter's Child and Adolescent Psychiatry. Massachusetts: Blackwell publishing; 2008. p. 930-945.

3. Merikangas KR, He JP, Burstein M, Swendsen J, Avenevoli S, Case B, et al. Service utilization for lifetime mental disorders in U.S. Adolescents: Results of the National Comorbidity Survey-Adolescent Supplement (NCSA). J Am Acad Child Adolesc Psychiatry 2011; 50: 32-45.

4. Merikangas KR, He JP, Burstein M, Swanson SA, Avenevoli S, Cui L, et al. Lifetime prevalence of mental disorders in U.S. adolescents: results from the National Comorbidity Survey Replication - Adolescent Supplement. J Am Acad Child Adolesc Psychiatry 2010; 49: 980-989.

5. Egger HL, Costello EJ, Erkanli A, Angold A. Somatic complaints and psychopathology in children and adolescents: Stomach aches, muscular-skeletal pains and headaches. J Am Acad Child Adolesc Psychiatry 1999; 38: 852-860.

6. Myers K, Winters NC. Ten-Year Review of Rating Scales. I: Overview of Scale functioning, psychometric properties and selection. J Am Acad Child Adolesc Psychiatry $2002 ; 41: 114-122$.

7. Holmbeck GN, Thill AW, Bachanas P, Garber J, Miller $\mathrm{KB}$, Abad M, et al. Evidence-based Assessment in pediatric psychology: Measures of psychosocial adjustment and psychopathology. J Ped Psychol. 2008; 33: 958-980.

8. Saunders CD, Hall EJ, Casey JE, Strang JD. Subtypes of Psychopathology in Children Reffered for Neuropsychological Assessment. Child Neuropsychol 2000; 6: 129-143.

9. Wirt RD, Lachar D, Klinedinst J, Seat PD. Multidimensional description of child personality: A manual for Personality Inventory for Children (Revision by David Lachar). Los Angeles: Western Psychological Services; 1990.

10. Lachar D. Personality Inventory for Children (PIC). Revised Format Manual Supplement. Los Angeles: Western Psychological Services; 1982.

11. Roebuck TM, Mattson S, Riley EP. Behavioral and psychosocial profiles of alcohol exposed children. Alcohol Clin Exp Res 1999; 23: 1070-1076.

12. Kelly EJ, Van Vactor JC. Distinguishing between conduct problem and emotionally disturbed students in secondary school: a five-instrument discriminant analysis. Psychol Rep 1991; 69: 303-311.

13. Kelly EJ, Van Vactor JC. Distinguishing between conduct-problem and emotionally disturbed students in elementary school: a five-instrument discriminant analysis. Psychol Rep 1992; 70: 311-319.

14. Costello EJ, Mustillo S, Erkanli A, Keeler G, Angold A. Prevalence and development of psychiatric disorderes in childhood and adolescence. Arch Gen Psychiatry 2003; 60: 837-844.

15. Ford T, Goodman R, Meltzer H. The british child and adolescent mental health survey 1999: The prevalence of DSM-IV disorders. J Am Acad Child Adolesc Psychiatry 2003; 42(10): 1203-1211.

16. Greene RW, Biederman J, Zerwas S, Monuteaux MC, Goring JC, Faraone SV. Psychiatric comorbidity, Family Dysfunction and Social Impairment in Reffered Youth with Oppositional Defiant Disorder. Am J Psychiatry 2002; 159: 1214-1224.

17. Nordahl HM, Ingul JM, Nordvik H, Wells A. Does Maternal Psychopathology Discriminate Between Children with DSM-IV Generalized Anxiety Disorder or Oppositional Defiant Disorder? The Predictive Validity of Maternal Axis I and Axis II Psychopathology. Eur Child Adolesc Psychiatry 2007; 16: 87-95.

18. Nordahl HM, Wells A, Olsson CA, Bjerkeset O. Association between abnormal psychosocial situations in childhood, generalized anxiety disorder and oppositional defiant disorder. Aus N Z J Psychiatry 2010; 44: 852-858.

19. American Psychiatric Association. Diagnostic and Statistical Manual of Mental Disorders (DSM-IV). Washington (DC): American Psychiatric Association; 1994. 
20. Tabachnik BG, Fidell LS. Using Multivariate Statistics, 5th edn. Boston: Pearson International; 2007.

21. Strauss CC, Last CG, Hersen M, Kazdin AE. Association between anxiety and depression in children and adolescents with anxiety disorders. J Abnorm Child Psychol 1988; 16: 57-68.

22. Warren SL, Sroufe LA. Developmental Issues. In: O1lendick TH, March JS, eds. Phobic and Anxiety Disorders in Children and Adolescents. New York: Oxford University Press; 2004. p. 92-116.

23. Dadds MR, James RC, Barrett PM, Verhulst FC. Diagnostic Issues. In: Ollendick TH, March JS, eds. Phobic and Anxiety Disorders in Children and Adolescents. New York: Oxford University Press; 2004. p. 3-33.

24. Biederman J, Faraone SV, Mick E, Moore P, Lelon E. Child Behavior Checklist findings further support comorbidity between ADHD and major depression in a referred sample. J Am Acad Child Adolesc Psychiatry 1996; 35: 734-742.
25. Engel NA, Rodrigue JR, Geffken GR. Parent-Child agreement on ratings of anxiety in children. Psychol Rep 1994; 5: 1251-1260.

26. Achenbach TM. Manual for the child behavior checklist 14-18 and 1991 profile. Burlington, VT: University of Vermont, Department of psychiatry; 1991.

27. Pearson DA, Lachar D. Using behavioural questionnaires to identify adaptive deficits in elementary school children. J Sch Psychol 1994; 32: 33-52.

\author{
Corresponding author: \\ Jo Magne Ingul \\ Department of Child and Adolescent Psychiatry \\ Levanger Hospital, Kirkegt 2 \\ 7600 Levanger, Norway \\ Phone: +47 74098955 \\ Fax: + 4774098329 \\ E-mail: jo.magne.ingul@hnt.no
}

